\title{
Evaluation of Optimum Instrument Conditions for the Best Spatial Resolution in Atomic-Column X-ray Analysis toward Quantification
}

\author{
Masashi Watanabe \\ Lehigh University, Bethlehem, Pennsylvania, United States
}

Improvement of spatial resolution in imaging and analysis is one of the primary objectives to utilize aberration-corrected (scanning) transmission electron microscopes ((S)TEMs). Both in STEM and TEM imaging, sub- $\AA$ resolution is routinely available in such aberration-corrected instruments. Especially in STEM imaging, the latest aberration-corrected microscopes ultimately, offer below half $\AA$ resolution [1]. The aberration correction for STEMs offers more probe currents while maintaining refined probe sizes. Therefore, both spatial resolution and sensitivity for analysis can be significantly improved and atomicresolution elemental mapping is now regularly carried out by both electron energy-loss spectrometry (EELS) and X-ray energy dispersive spectrometry (XEDS) in the latest instruments [e.g. 2]. Obviously, the aberration correction has brought further flexibility in instrumental conditions and it is very important to configure those operating conditions to achieve the best possible spatial resolution in analysis. This study explores theoretically the optimum instrumental conditions in terms of (i) the convergence semiangle $(\alpha)$ and (ii) the defocus $(\Delta z)$ for the best spatial resolution in atomic-column X-ray analysis, which should be applicable to EELS analysis as well.

For evaluating the optimum conditions, the X-ray signal simulation scheme developed for an oriented crystalline specimen [3] was used. This approach is based on the multislice calculation for electron propagation (wave function) via xHREM software package [4] in combination with X-ray spectrum simulation through legacy Desktop Spectrum Analyzer (DTSA) codes [5]. Figure 1 shows cross-section views ([010] projection) of wave functions at a Ga position in a [001]-oriented GaAs specimens at the convergence semi-angle $(\alpha)$ of 11,25 and $35 \mathrm{mrad}$. Note that results at 25 and $35 \mathrm{mrad}$ are under aberration correction but not at $11 \mathrm{mrad}$, and the incident probe size decreases as $\alpha$ increases. These wave functions were calculated at the probe current of $250 \mathrm{pA}$ (a typical value for atomic-column mapping). At each sliced plane, the beam broadening size, which contains $90 \%$ of the incident intensity (a typical definition for X-ray analysis, e.g. [6]), was determined by central limit integration, and plotted against the specimen thickness in Fig. 2. This beam broadening size can be treated as the spatial resolution in analysis, and traditional Gaussian beam broadening model [7] was also plotted in Fig. 2 for comparison. At very thin region below $5 \mathrm{~nm}$, the smallest broadening can be obtained at $\alpha=35 \mathrm{mrad}$. However, with an increase of the specimen thickness, broadening is less at lower $\alpha$. So, higher convergence angles offered by the aberration correction enhances the broadening at thicker regions. The Gaussian broadening model shows the least broadening due to lack of the convergence-angle term.

Using this simulation approach, X-ray maps can also be generated. Figure 3 shows a set of Ga intensity ratio maps (the $\mathrm{Ga} \mathrm{K} \alpha$ intensity over sum of the $\mathrm{Ga} \mathrm{K} \alpha$ and $\mathrm{As} \mathrm{K} \alpha$ intensities, roughly equivalent to the $\mathrm{Ga}$ concentration fraction) of the [001]-projected GaAs unit cell at different $\alpha$ and different specimen thicknesses. The maps at $\alpha=11 \mathrm{mrad}$ show poor resolution and the Ga ratio does not reach zero and 1 at the As and Ga positions, respectively. Obviously, the fine incident probe size, i.e. the aberration correction really matters to achieve atomic resolution although the beam broadening is less at thicker regions. In the aberration-corrected conditions at $\alpha=25$ and $35 \mathrm{mrad}$, superior resolution can be obtained up to the 40$\mathrm{nm}$-thick specimen. At the specimen thickness is very thin $(\sim 5 \mathrm{~nm})$, the Ga ratio reaches zero and 1 at the 
As and Ga positions, respectively, both at $\alpha=25$ and $35 \mathrm{mrad}$. However, the resolution of the Ga ratio map is better at $\alpha=25 \mathrm{mrad}$ when thicker specimens are analysed.

The Ga ratio maps shown in Fig. 3 were simulated in the conditions that the incident probe is focused on the top surface (incident probe side). Figure 4 shows a set of the Ga ratio maps at $\alpha=35 \mathrm{mrad}$ simulated when the focal point is shifted to inside of the specimen. When the defocus amount is small, then better spatial resolution is maintained. In general, however, the defocus degrades the spatial resolution and quantification. Therefore, the focal point should be kept at the top surface, i.e. the focal position for the best annular dark-field imaging in STEMs.[8]

Fig. 1

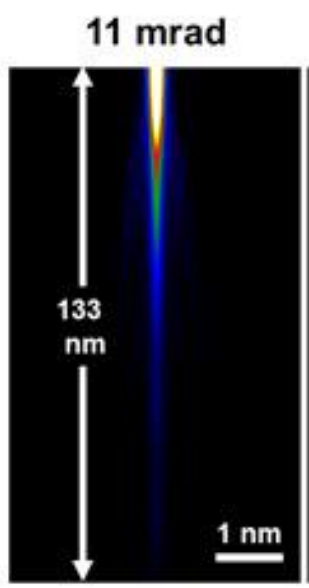

Ga column

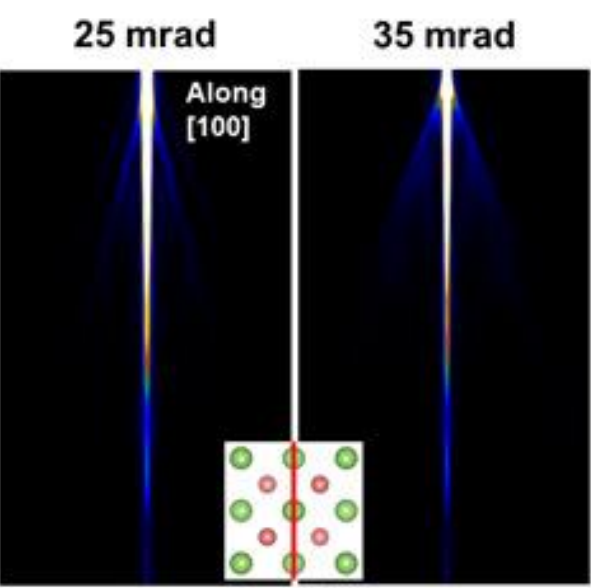

Fig. 3

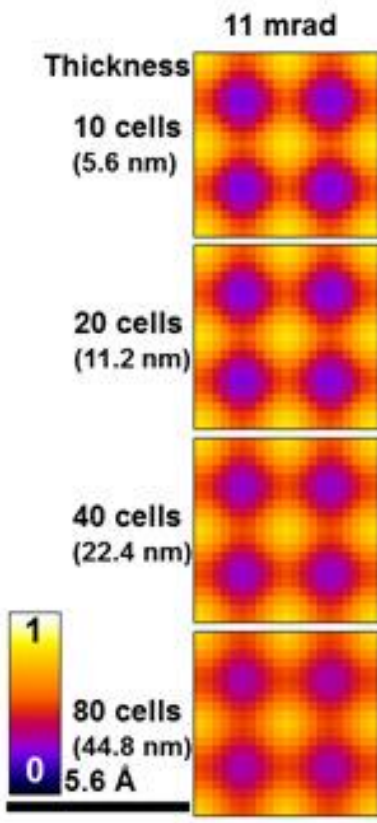

Convergence angle

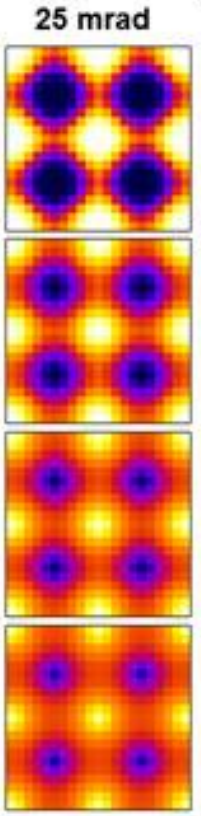

$35 \mathrm{mrad}$

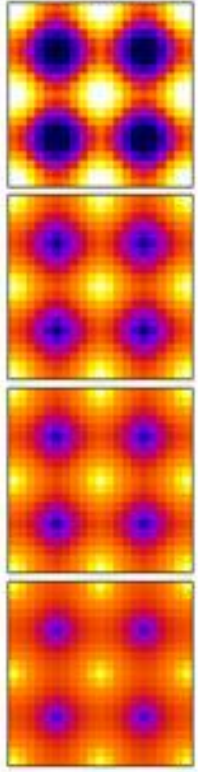

Fig. 2

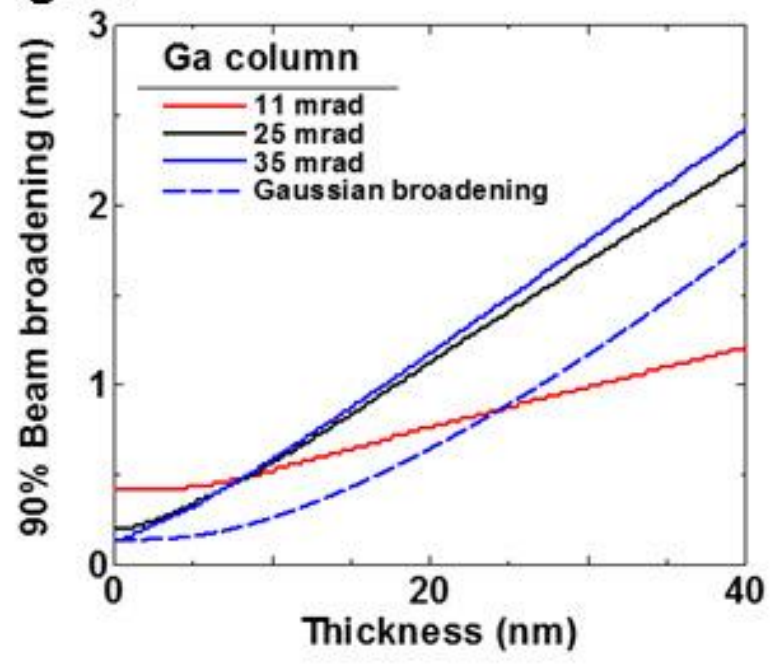

Fig. 4

Probe focal point (35 mrad)

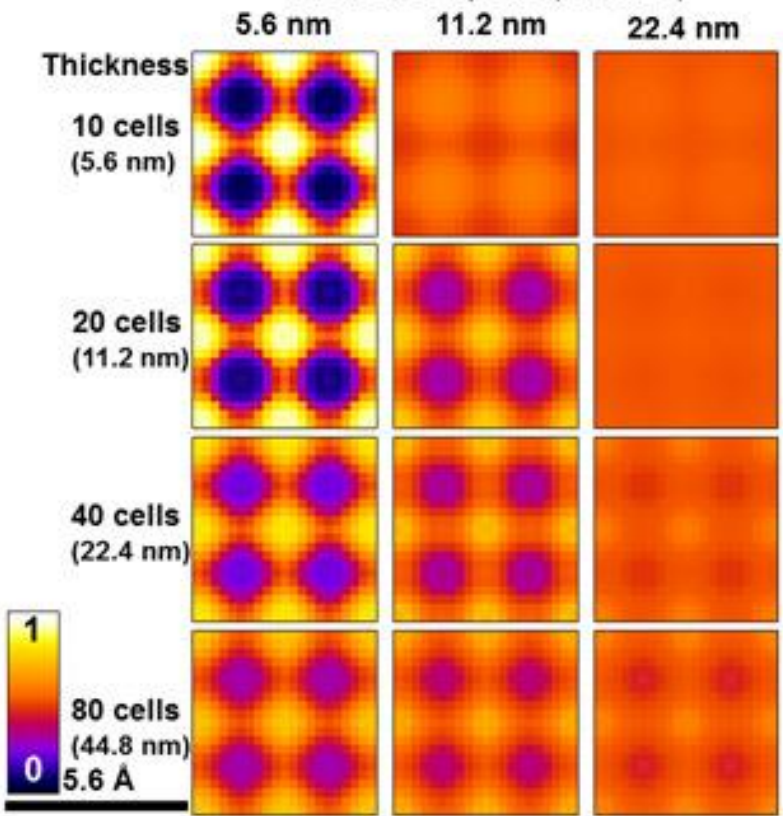

Figure 1. Figure 1: Wave functions simulated at a Ga position in a [001]-oriented GaAs specimens at $\alpha=$ 11, 25 and $35 \mathrm{mrad}$. Figure 2: The probe broadening size with $90 \%$ of the incident intensity determined 
from the simulated wave functions, plotted as a function of the specimen thickness. Figure 3: A set of the Ga ratio maps of the [001]-projected GaAs unit cell summarized at different $\alpha$ and the different specimen thicknesses. Figure 4: A set of the Ga ratio maps of the [001]-projected GaAs unit cell simulated at $\alpha=35 \mathrm{mrad}$ and the different focal position.

References

[1] H. Sawada et al, Microsc. Miroana. 20 (2014), Suppl. 3, 124.

[2] S.J. Pennycook \& P.D. Nellist ed. Scanning Transmission Electron Microscopy: Imaging and Analysis, Springer, NY, (2011).

[3] M. Watanabe, Microsc. Miroana. 21 (2015), Suppl. 3, 1081.

[4] K. Ishizuka., J. Electron Microsc. 50 (2012), 291.

[5] C.E. Fiori et al. Public domain DTSA software package (1992).

[6] D.B. Williams \& C.B. Carter. Transmission Electron Microscopy, $2^{\text {nd }}$ ed. Springer, NY (2009).

[7] P. Doig et al, Philos. Mag. A, 41 (1080), 761.

[8] The author wishes to acknowledge financial support from the NSF through grants DMR-2018683 and CMMI-2016279. 